\title{
Reversible Vortex Ratchet Effects and Ordering in Superconductors with Simple Asymmetric Potential Arrays
}

\author{
Qiming $\mathrm{Lu}^{1,2}$, C.J. Olson Reichhardt ${ }^{1}$, and C. Reichhardt ${ }^{1}$ \\ ${ }^{1}$ Center for Nonlinear Studies and Theoretical Division, \\ Los Alamos National Laboratory, Los Alamos, New Mexico 87545 \\ ${ }^{2}$ Department of Physics, Applied Physics, 8 Astronomy, \\ Rensselaer Polytechnic Institute, Troy, New York, 12180-3590
}

(Dated: September 16, 2018)

\begin{abstract}
We demonstrate using computer simulations that the simplest vortex ratchet system for type-II superconductors with artificial pinning arrays, a simple asymmetric potential array, exhibits the same features as more complicated two-dimensional (2D) vortex ratchets that have been studied in recent experiments. We show that the simple geometry, originally proposed by Lee et al. [Nature 400, 337 (1999)], undergoes multiple reversals in the sign of the ratchet effect as a function of vortex density, substrate strength, and ac drive amplitude, and that the sign of the ratchet effect is related to the type of vortex lattice structure present. Thus, although the ratchet geometry has the appearance of being effectively $1 \mathrm{D}$, the behavior of the ratchet is affected by the $2 \mathrm{D}$ structure of the vortex configuration. When the vortex lattice is highly ordered, an ordinary vortex ratchet effect occurs which is similar to the response of an isolated particle in the same ratchet geometry. In regimes where the vortices form a smectic or disordered phase, the vortex-vortex interactions are relevant and we show with force balance arguments that the ratchet effect can reverse in sign. The dc response of this system features a reversible diode effect and a variety of vortex states including triangular, smectic, disordered and square.
\end{abstract}

PACS numbers: $74.25 . \mathrm{Qt}$

\section{INTRODUCTION}

When an overdamped particle is placed in an asymmetric potential and an additional ac drive is applied, a net dc drift velocity or rectification can occur which is known as the ratchet effect. Stochastic ratchets can be constructed with Brownian particles, while ordinary ratchets can be created in deterministic systems [1]. Typically, an applied ac drive or periodic flashing of the potential couples with some form of asymmetry in the substrate, breaking the symmetry of the particle motion. Ratchet effects have been studied in the context of molecular motors [1] as well as in the motion of colloidal particles [2], granular materials [3] and cold atoms in optical trap arrays [4].

Vortices in type-II superconductors act as overdamped particles moving in nanostructured potential landscapes. The possibility of realizing a ratchet effect to transport superconducting vortices was originally proposed by Lee et al. [5], who studied vortices interacting with a simple asymmetric or sawtooth substrate potential and an additional external ac drive. This type of asymmetric potential could be fabricated by creating a superconducting sample that has an asymmetric thickness modulation. The external ac drive is provided by either an oscillating external magnetic field or by an applied ac current which produces an oscillating Lorentz force on the vortices. The numerical simulations of Ref. [5] indicated that a net dc motion of vortices could arise upon application of an ac drive, and that the net vortex drift is in the easy direction of the asymmetric substrate, as would be expected for a single particle interacting with such a potential. The original work on vortex ratchets was followed by a series of proposals for alternative vortex ratchets based on 2D asymmetric pinning array geometries, each of which produces similar types of vortex ratchet effects with the net vortex flow in the easy direction of the underlying potential [6, 7, 8, 9, 10]. These ratchets operate either through a bottleneck effect [6, 9] or by means of an effectively 1D mechanism [7, 8, 10]. Similar ratchet effects have also been found for superconductors with asymmetric surface barriers [11].

Recently, new types of 2D periodic pinning geometries have been proposed that exhibit not only the regular ratchet effect but also a reverse ratchet effect, in which the net dc vortex motion is in the hard substrate direction 12, 13, 14, 15, 16, 17]. In some cases, ratchet reversals occur when the vortex-vortex interactions at higher fillings become relevant, resulting in an effective asymmetric potential oriented in the direction opposite to the pinning structure. As a result, some of the vortices move in the hard direction of the pinning substrate, and the net motion is described by either an effectively 1D [14, 15, 17] or 2D [16] mechanism. Other vortex ratchet systems have multiple sign reversals due to symmetry breaking from multiple circular ac drives [12] or three state pinning potentials [13]. The reverse vortex ratchet effect has been observed in experiments with $2 \mathrm{D}$ arrays of triangular pinning sites [15] and other types of 2D arrays [17]. In the work of Silva et al. [17], the periodic pinning array in the experimental sample has a two component unit cell containing a large pinning site with a small pinning site placed to one side. This system exhibits a remarkable field dependent multiple reverse ratchet effect. For the first matching field and higher odd matching fields, a 


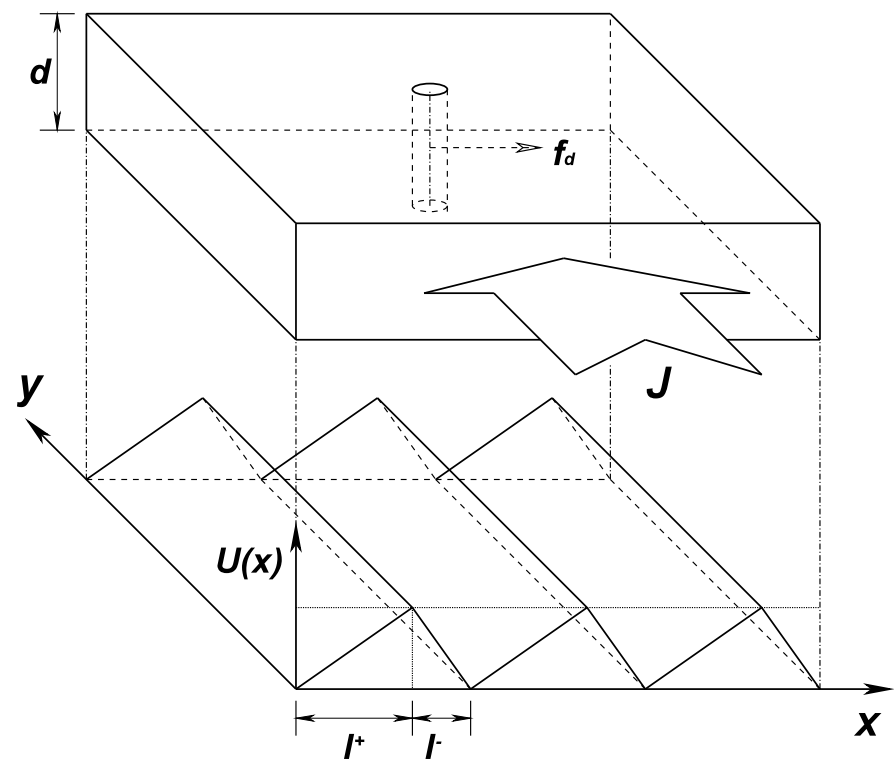

FIG. 1: Schematic of the simulation geometry. Vortices are subjected to an applied current $\mathbf{J}=J \hat{\mathbf{y}}$ which produces a driving force $\mathbf{F}^{e x t}=F_{d c} \hat{\mathbf{x}}$ or $F_{a c} \hat{\mathbf{x}}$, illustrated in the top portion of the figure. The bottom portion of the figure shows the periodic potential $U(x)$ through which the vortices move. The width of the easy direction of the pinning potential is $l^{+}$ and the width of the hard direction is $l^{-}$, where $l^{+}>l^{-}$and $l^{+}+l^{-}=a$, the pinning lattice constant.

regular ratchet effect occurs, but at even matching fields the ratchet effect changes sign. Simulations indicate that the pinning sites are capturing multiple vortices, and that this can change the direction of the asymmetric effective potential at every matching field, as described by a 1D model. A ratchet effect has also been observed in a Josephson-junction array structure which has spatial asymmetry [18]. Here, a diode effect occurs where the depinning field is higher in the hard direction. A ratchet and reversed ratchet effect are also seen for matching fields less than one.

In this work, we perform a detailed investigation of the simplest vortex ratchet system created by an asymmetric periodic modulation, which was originally shown to exhibit only an ordinary ratchet effect [5]. By considering magnetic fields much higher than those used in Ref. [5], where the effectively single vortex regime was studied, we show that this system can also exhibit ratchet sign reversals as a function of vortex density when the vortex interactions become relevant. Unlike the majority of previously studied vortex ratchets, the behavior of this system is determined by the detailed $2 \mathrm{D}$ structure of the vortex configuration, and cannot be explained in terms of a simple 1D model. We find that the dc response exhibits a reversible diode effect correlated with the regions where the ratchet effect reverses. The sign of the ratchet effect is shown to be related to the nature of the overall vortex lattice structure. At magnetic field densities where the

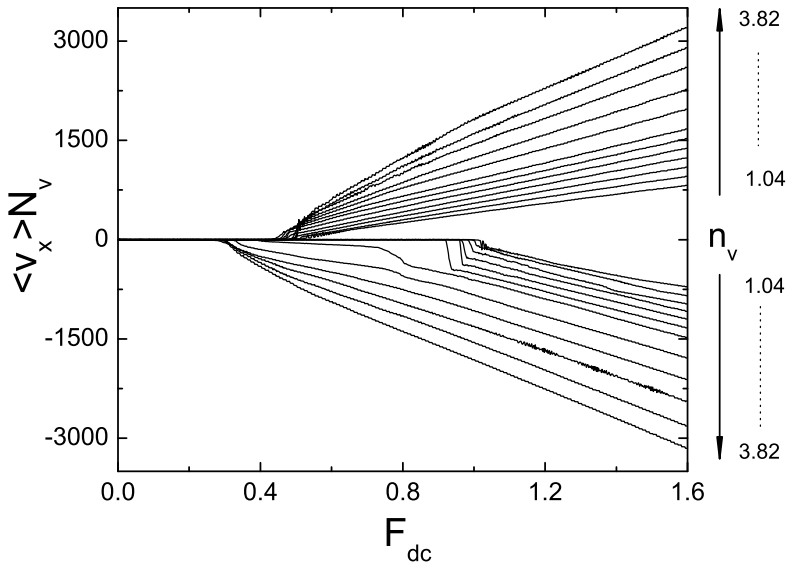

FIG. 2: The dc non-normalized average velocity $\left\langle V_{x}\right\rangle N_{v}$ vs $F_{d c}$ for varied $n_{v}$ and both directions of dc drive. The curves for $\mathbf{F}_{i}^{e x t}=\hat{\mathbf{x}} F_{d c}$ have $\left\langle V_{x}\right\rangle N_{v} \geq 0$, while the curves for $\mathbf{F}_{i}^{e x t}=-\hat{\mathbf{x}} F_{d c}$ have $\left\langle V_{x}\right\rangle N_{v} \leq 0$. In order of increasing $\left|\left\langle V_{x}\right\rangle N_{v}\right|$, the curves have $n_{v}=1.04 / \lambda^{2}, 1.22 / \lambda^{2}, 1.39 / \lambda^{2}$, $1.56 / \lambda^{2}, 1.74 / \lambda^{2}, 1.91 / \lambda^{2}, 2.08 / \lambda^{2}, 2.43 / \lambda^{2}, 2.78 / \lambda^{2}, 3.13 / \lambda^{2}$, $3.47 / \lambda^{2}$, and $3.82 / \lambda^{2}$. For $n_{v}<2.08 / \lambda^{2}$, the negative drive critical depinning force is larger than the positive drive critical depinning force, $f_{c}^{-}>f_{c}^{+}$, but for $n_{v} \geq 2.08 / \lambda^{2}$, this reverses and $f_{c}^{-}<f_{c}^{+}$.

vortex lattice has a more ordered or crystalline structure, the effective vortex interactions cancel or are reduced by the symmetry of the lattice, and the response is the ordinary ratchet effect as in the case of a single vortex. At fields where the vortex lattice is disordered or has an intrinsic asymmetry, the interactions between individual vortices become relevant and a portion of the vortices move in the easy direction of the pinning potential, resulting in a reversal of the sign of the ratchet effect which can be predicted from force balance arguments. In addition to the ratchet response of this system, we show that a rich variety of distinct vortex phases can be realized as a function of density, including crystalline, smectic, disordered and square phases. Commensuration effects also create oscillations in the critical depinning force which are distinct from those observed for two dimensional periodic pinning arrays.

\section{SIMULATION}

We consider a 2D system of size $L_{x}=L_{y}=24 \lambda$, where $\lambda$ is the London penetration depth, with periodic boundary conditions in the $x$ and $y$ directions. The sample contains $N_{v}$ vortices interacting with an asymmetric substrate $U(x)$ of period $a=\lambda$, illustrated schematically in Fig. 1. The vortex density $n_{v}=N_{v} / L_{x} L_{y}$. A given 
vortex $i$ obeys the overdamped equation of motion

$$
\eta \frac{d \mathbf{R}_{i}}{d t}=\mathbf{F}_{i}^{v v}+\mathbf{F}_{i}^{s}+\mathbf{F}_{i}^{e x t}+\mathbf{F}_{i}^{T} .
$$

The damping constant $\eta=\phi_{0}^{2} d / 2 \pi \xi^{2} \rho_{N}$, where $d$ is the sample thickness, $\xi$ is the coherence length, $\rho_{N}$ is the normal-state resistivity, and $\phi_{0}=h / 2 e$ is the elementary flux quantum. The vortex-vortex interaction force is

$$
\mathbf{F}_{i}^{v v}=\sum_{j \neq i}^{N_{v}} f_{0} K_{1}\left(\frac{r_{i j}}{\lambda}\right) \hat{\mathbf{r}}_{i j}
$$

Here $r_{i j}=\left|\mathbf{r}_{i}-\mathbf{r}_{j}\right|, \hat{\mathbf{r}}_{i j}=\left(\mathbf{r}_{i}-\mathbf{r}_{j}\right) / r_{i j}$, and $\mathbf{r}_{i(j)}$ is the position of vortex $i(j)$. Force is measured in units of $f_{0}=\phi_{0}^{2} / 2 \pi \mu_{0} \lambda^{3}$, and time in units of $\tau=\eta / f_{0}$. $K_{1}\left(r_{i j} / \lambda\right)$ is the modified Bessel function which falls off exponentially for $r_{i j}>\lambda$. For computational efficiency, the vortex-vortex interaction force is cut off at $6 \lambda$. We have previously found that using longer cutoff lengths produces negligible effects [20]. The pinning potential is modeled as a sawtooth as shown in Fig. 1.

$$
\mathbf{F}_{i}^{s}= \begin{cases}-\frac{1}{2} A_{p} \hat{\mathbf{x}} & \text { if } 0 \leq x_{i} \bmod a<l^{+} \\ A_{p} \hat{\mathbf{x}} & \text { if } l^{+} \leq x_{i} \bmod a<a .\end{cases}
$$

Here, $A_{p}=1.0 f_{0}$ and $x_{i}=\mathbf{r}_{i} \cdot \hat{\mathbf{x}}$. The width of the long or "easy" side of the pinning potential is $l^{+}=(2 / 3) a$ and the width of the short or "hard" side is $l^{-}=(1 / 3) a$ such that $l^{+}+l^{-}=a$. The term $\mathbf{F}_{i}^{e x t}$ in Eq. 1 is the force from a dc or ac external driving current. For the case of an applied dc drive, $\mathbf{F}_{i}^{e x t}=F_{d c} \hat{\mathbf{x}}$, where $F_{d c}$ is increased from zero in increments of $\delta F_{d c}=0.01$ every $10^{4}$ simulation time steps. For the case of an applied ac drive, $\mathbf{F}_{i}^{e x t}= \pm F_{a c} \hat{\mathbf{x}}$, where the positive sign is used during the first half of each period $\tau$ and the negative sign is used during the second half period, resulting in a square wave centered about zero. The initial vortex configurations are obtained through simulated annealing, where we set $\mathbf{F}_{i}^{e x t}=0$ and apply a thermal force with the properties $\left\langle\mathbf{F}_{i}^{T}\right\rangle=0$ and $\left\langle\mathbf{F}_{i}^{T}(t) \mathbf{F}_{j}^{T}\left(t^{\prime}\right)\right\rangle=2 \eta k_{B} T \delta_{i j} \delta\left(t-t^{\prime}\right)$. The simulated annealing begins at a high temperature $F^{T}=5.0$ which is well above the melting temperature of the vortex lattice, and then $F^{T}$ is slowly reduced to zero. After annealing, we set $\mathbf{F}_{i}^{T}=0$, apply a dc or ac drive, and measure the average velocity $\left\langle V_{x}\right\rangle=\left\langle\left(N_{v}^{-1}\right) \sum_{i=0}^{N_{v}} \mathbf{v}_{i} \cdot \hat{\mathbf{x}}\right\rangle$. For dc driving, we identify the critical currents in the positive and negative driving directions, $f_{c}^{+}$and $f_{c}^{-}$, which we define as the drive at which $\left|\left\langle V_{x}\right\rangle\right|=0.1 f_{0}$.

\section{DC DEPINNING AND VORTEX LATTICE STRUCTURES}

We first compare the dc response of the system for driving in the positive (easy) and negative (hard) $x$ direction. The top set of curves in Fig. 2 show $\left\langle V_{x}\right\rangle N_{v}$

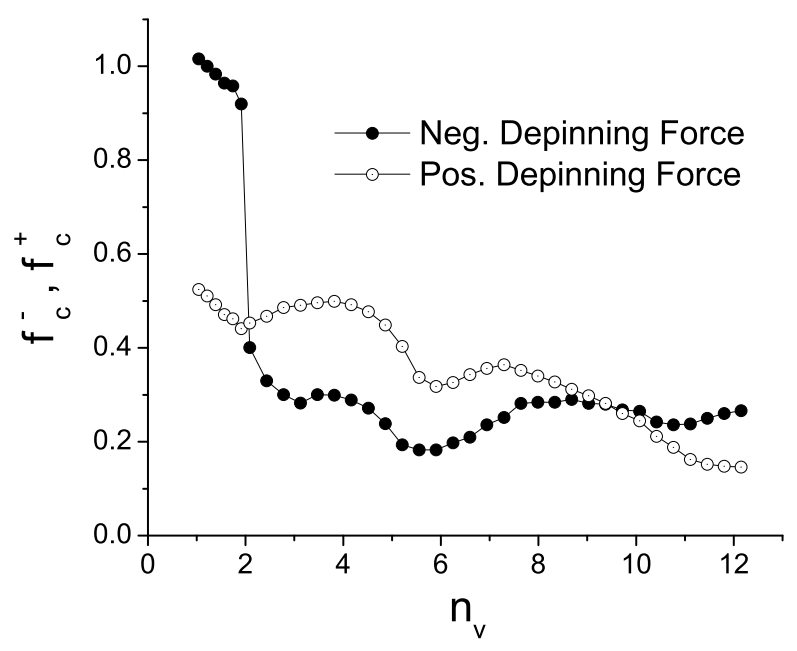

FIG. 3: The depinning force curves vs $n_{v}$ for the system in Fig. 2 for driving in the negative direction, $f_{c}^{-}$(black circles), and in the positive direction, $f_{c}^{+}$(open circles). The diode effect reverses near $n_{v}=1.91 / \lambda^{2}$ and $n_{v}=9.90 / \lambda^{2}$. Several oscillations in the depinning force also occur.

versus $F_{d c}$ for $\mathbf{F}_{i}^{e x t}=\hat{\mathbf{x}} F_{d c}$ for samples with $N_{v}$ ranging from $n_{v}=1.04 / \lambda^{2}$ to $3.82 / \lambda^{2}$. The bottom set of curves show $\left\langle V_{x}\right\rangle N_{v}$ versus $F_{d c}$ for the same samples with $\mathbf{F}_{i}^{e x t}=-\hat{\mathbf{x}} F_{d c}$. A clear diode effect occurs for $n_{v}<2.08 / \lambda^{2}$ when the negative (hard) critical depinning force $f_{c}^{-}$is larger than the positive (easy) critical depinning force $f_{c}^{+}$. Here, $f_{c}^{-} / f_{0} \lesssim 1$, close to the value of the pinning force in the hard direction, while $f_{c}^{+} / f_{0} \approx 0.5$, nearly the same as the easy direction pinning force. Thus, for $n_{v}<2.08 / \lambda^{2}$, the depinning force in each direction is primarily determined by the pinning substrate and the vortex-vortex interactions are only weakly relevant. A pronounced drop in $f_{c}^{-}$from $f_{c}^{-} / f_{0} \approx 1$ to $f_{c}^{-} / f_{0} \approx 0.4$ occurs above $n_{v}=2.08 / \lambda^{2}$. For $n_{v}>2.08 / \lambda^{2}$, the diode effect is reversed since now $f_{c}^{-}<f_{c}^{+}$.

When $n_{v}=2.08 / \lambda^{2}$, the negative depinning curve in Fig. 2 shows a clear two step depinning process with an initial depinning near $F_{d c}=0.45 f_{0}$ followed by a second jump in $\left\langle V_{x}\right\rangle$ near $F_{d c}=0.8 f_{0}$. At the low first depinning threshold, only a portion of the vortices depin, while at the second threshold near $F_{d c}=0.8 f_{0}$, the entire lattice depins.

The values of $f_{c}^{+}$and $f_{c}^{-}$undergo changes as a function of $n_{v}$ that are difficult to discern in Fig. 2, In Fig. 3 we plot both $f_{c}^{+}$and $f_{c}^{-}$versus $n_{v}$ for systems with up to 7000 vortices and $n_{v}=12.15 / \lambda^{2}$. There is a positive diode effect for $n_{v}<1.91 / \lambda^{2}$ when $f_{c}^{-}>f_{c}^{+}$. The diode effect reverses for $1.91 / \lambda^{2} \leq n_{v} \leq 9.90 / \lambda^{2}$, where $f_{c}^{-}<f_{c}^{+}$. A second reversal occurs for $n_{v}>9.90 / \lambda^{2}$, when $f_{c}^{+}$drops back below $f_{c}^{-}$. The high $n_{v}$ reversal is shown in more detail in Fig. 4, where we plot $\left\langle V_{x}\right\rangle$ versus $F_{d c}$ for two different samples. In Fig. $4(\mathrm{a})$, 

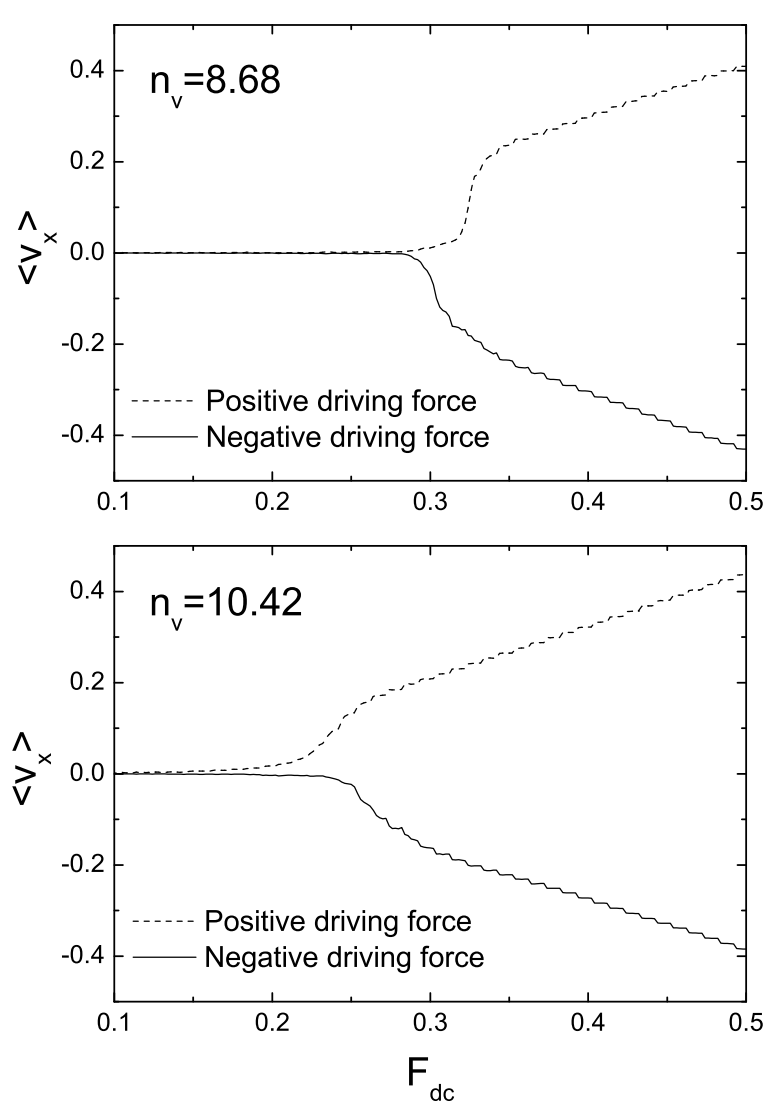

FIG. 4: $\left\langle V_{x}\right\rangle$ versus $F_{d c}$ curves for positive driving, $\mathbf{F}_{i}^{e x t}=$ $\hat{\mathbf{x}} F_{d c}$ (upper dashed line), and negative driving, $\mathbf{F}_{i}^{e x t}=-\hat{\mathbf{x}} F_{d c}$ (lower solid line). (a) A sample with $n_{v}=8.68 / \lambda^{2}$ showing a negative diode effect with $f_{c}^{-}<f_{c}^{+}$. (b) A sample with $n_{v}=10.42 / \lambda^{2}$ showing a positive diode effect with $f_{c}^{-}>f_{c}^{+}$.

at $n_{v}=8.68 / \lambda^{2}, f_{c}^{-}<f_{c}^{+}$and we find a negative diode effect. The situation is reversed in Fig. 4(b) at $n_{v}=10.42 / \lambda^{2}$, where $f_{c}^{-}>f_{c}^{+}$and a positive diode effect occurs.

Fig. 3 indicates that $f_{c}^{+}$and $f_{c}^{-}$both undergo oscillations. A simple 1D picture for the vortex structure would predict maxima to appear in the depinning force at the commensurate fields at which an integer number of vortex rows fit inside each pinning trough, given by $n_{v}=n^{2} / \lambda^{2}$, where $n$ is an integer. We do observe a maximum in both $f_{c}^{+}$and $f_{c}^{-}$near $n_{v}=4.0 / \lambda^{2}$, corresponding to the presence of two ordered commensurate vortex rows per channel. Although $f_{c}^{-}$has a very broad plateau centered around $n_{v}=9.0 / \lambda^{2}$ which could be interpreted as the $n=3$ matching field, the inadequacy of a simple $1 \mathrm{D}$ picture is indicated by the fact that for $f_{c}^{+}$, the corresponding maximum falls at the much lower field $n_{v}=7.81 / \lambda^{2}$. For vortex densities just above and below each of the commensurate fields, the addition or subtraction of a small number of vortices does not change the number of rows in each trough but instead causes a disordering of the rows relative to one another, resulting in a smectic structure. This occurs because the shear modulus is smaller than the compression modulus of the rows. As larger numbers of vortices are added, the compression of each row grows until the rows begin to buckle. The buckling transition destroys the alignment perpendicular to the vortex rows, causing the vortex configuration to become more isotropically disordered. This is similar to the commensuration effects found for vortex lattices in multilayer systems [21]. In this case, the field is applied along the direction of the layers so that the vortices effectively interact with a potential that is periodically modulated in one dimension. These experiments found oscillations in the magnetization, which is proportional to the depinning force. The oscillations were also attributed to transitions in the vortex lattice structure from $n$ chains per layer to $n+1$ chains per layer [21].

The vortex configuration for $n_{v}<1.04 / \lambda^{2}$ has only one column of vortices per pinning trough modulation, as illustrated in Fig. 5(a). The presence of triangular ordering in the vortex lattice is clearly indicated by the structure factor $S(\mathbf{k})$,

$$
S(\mathbf{k})=N_{v}^{-1}\left|\sum_{i=1}^{N_{v}} \exp \left(i \mathbf{k} \cdot \mathbf{r}_{i}\right)\right|^{2},
$$

plotted in Fig. 5(b). On average, the vortex-vortex interactions cancel along the $x$ direction since the vortices are equally spaced a distance $a$ apart in this direction. Near $n_{v}=2.0 / \lambda^{2}$, a transition in the vortex configuration occurs in which the vortex columns begin to buckle, as shown in Fig. 5(c). Here, the vortex-vortex interactions along the $x$ direction no longer cancel, and at points in the vortex columns where a buckled vortex is adjacent to the column, a vortex within the column experiences both the force from the pinning substrate, which has a maximum value of $A_{p}$, as well as an additional force from the buckled vortex, which has a maximum value of $K_{1}(a) f_{0}$. A simple estimate of the depinning force in a buckled region, $f_{c, b}^{-}$gives $f_{c, b}^{-}=A_{p}-K_{1}(a) f_{0}=0.395 f_{0}$, which is close to the value of $f_{c}^{-}$shown in Fig. 2 for $n_{v}>2.08 / \lambda^{2}$. For values of $n_{v}$ just above the buckling onset, buckling occurs in only a small number of places that are widely separated. Vortices near the buckled locations depin at $f_{c, b}^{-}$, while vortices outside of the buckled locations do not depin until $F_{d c}$ is close to $A_{p}$. The result is the two stage depinning process shown in Fig. 2 for $n_{v}=2.08 / \lambda^{2}$. As the vortex density is further increased above $n_{v}=2.08 / \lambda^{2}$, the distance between buckled regions decreases, smearing out the two step depinning process.

For $1.91 / \lambda^{2}<n_{v}<5.90 / \lambda^{2}, y$ direction spatial correlations in the vortex configurations are lost due to the buckling of the vortex columns, but the pinning substrate maintains the spatial correlations in the $x$ direction, resulting in smecticlike vortex structures. This is illustrated in Fig. 55(c,d) for $n_{v}=2.08 / \lambda^{2}$ and in Fig. 馬 $(\mathrm{e}, \mathrm{f})$ 


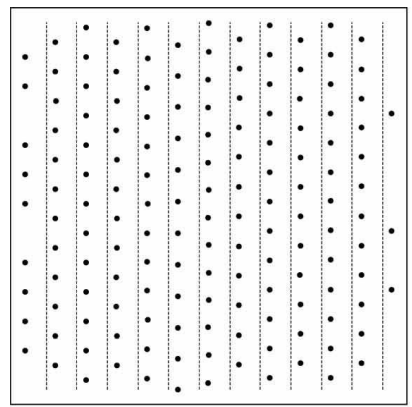

(a)

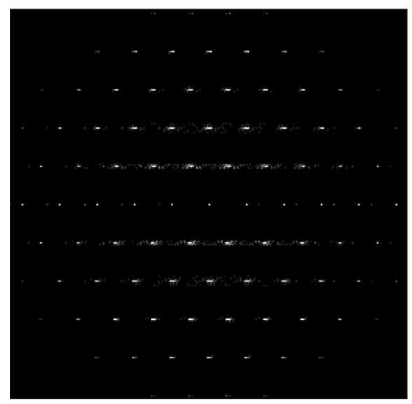

(b)

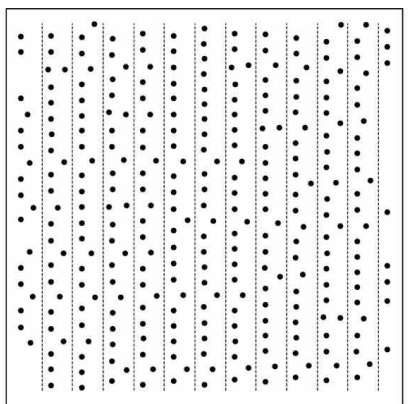

(c)

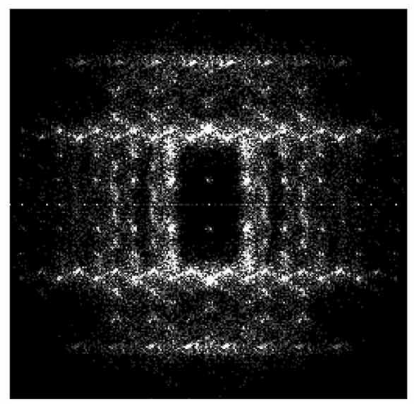

(d)

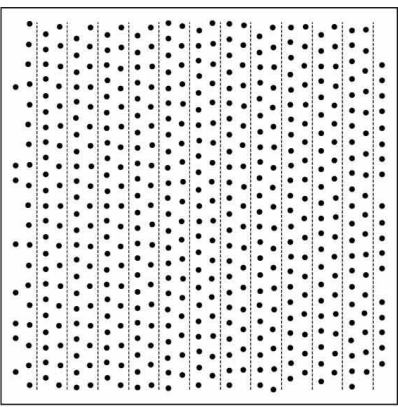

(e)

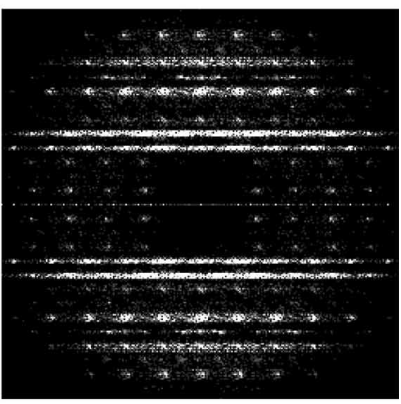

(f)

FIG. 5: (a) Vortex locations (black dots) and edges of the pinning troughs (black lines) in a $12 \lambda \times 12 \lambda$ subsection of a system with $n_{v}=1.04 / \lambda^{2}$ and no applied drive. A single column of vortices is confined within each trough. (b) Corresponding structure factor $S(\mathbf{k})$ for $n_{v}=1.04 / \lambda^{2}$. Here and in other plots of $S(\mathbf{k})$, we show a greyscale heightmap of $S\left(k_{x}, k_{y}\right)$ with white values higher than dark values, and with the origin of coordinates at the center of the panel. $k_{x}$ is along the vertical axis, and $k_{y}$ is along the horizontal axis. (c) Vortex and pinning trough locations for a system with $n_{v}=2.08 / \lambda^{2}$ and no applied drive. In some regions, the columns of vortices have buckled and a smectic type of ordering begins to appear. (d) $S(\mathbf{k})$ for $n_{v}=2.08 / \lambda^{2}$. (e) Vortex and pinning trough locations for a system with $n_{v}=3.47 / \lambda^{2}$ and no applied drive. Each trough contains two columns of vortices. (f) $S(\mathbf{k})$ for $n_{v}=3.47 / \lambda^{2}$. A double smectic ordering appears, as indicated by the two prominent lines in $S(\mathbf{k})$.

for $n_{v}=3.47 / \lambda^{2}$. At $n_{v}=2.08 / \lambda^{2}$ in Fig. 5 (c), a second column of vortices begins to form in each pinning trough by means of a buckling transition, as previously discussed. The corresponding structure factor in Fig. 5(d) shows some smectic type smearing, indicating that the vortices are less correlated in the $y$-direction than in the $x$-direction. As $N_{v}$ increases, the correlations in the $y$ direction are further decreased, as seen in $S(\mathbf{k})$ for $n_{v}=3.47 / \lambda^{2}$ in Fig. 5 (f). At this density, the vortex configuration shown in Fig. [5(e) indicates that there are two columns of vortices per trough, which is also reflected by the double band feature in $S(\mathbf{k})$. At $n_{v}=5.21 / \lambda^{2}$, shown in Fig. 6(a), the vortex configuration still has two columns of vortices in each pinning trough, and the two band feature in $S(\mathbf{k})$ is more prominent, as illustrated in Fig. 6(b). As $N_{v}$ increases further, the two column structure is destroyed and the vortex configuration becomes highly disordered in both the $x$ and $y$ directions, as seen at $n_{v}=6.94 / \lambda^{2}$ in Fig. 6 (c). Here $S(\mathbf{k})$ has a ring structure, as shown in Fig. 6 (d). The effect of the one-dimensional substrate can still be seen in the form of small modulations along $k_{x}$ in Fig. 6(d).

At $n_{v}=10.42 / \lambda^{2}$, plotted in Fig. 6(e), a new commensurate crystalline structure appears where four vortices fit across the pinning trough at a $45^{\circ}$ angle so that the overall vortex lattice symmetry is square. This is supported by the four prominent peaks that appear in $S(\mathbf{k})$ in Fig. 6(f). There is a competition between minimizing the vortex-vortex interaction energy, which favors a triangular lattice, and maximizing the vortex-substrate interaction, which favors commensurate vortex configurations. Since the difference in energy between a triangular and square vortex lattice is quite small, the commensuration effect is favored at $n_{v}=10.42 / \lambda^{2}$, and a square structure appears. Since the vortex lattice is symmetrical, the vortex-vortex interactions are strongly reduced and the depinning transition is similar to the case of $n_{v}<1.91 / \lambda^{2}$ where $f_{c}^{-}>f_{c}^{+}$and the vortices depin more readily in the easy direction. In this case, the depinning response is elastic, but since there are multiple vortex columns in each trough there is a pronounced distortion in the lattice when a dc force is applied, causing $f_{c}^{-}$to be significantly lower than $A_{p}$.

As a function of vortex density, the system thus passes through a sequence of triangular, smectic, disordered, and square vortex arrangements. These results show similarities to studies performed on magnetic particles interacting with simple symmetric periodic modulated 


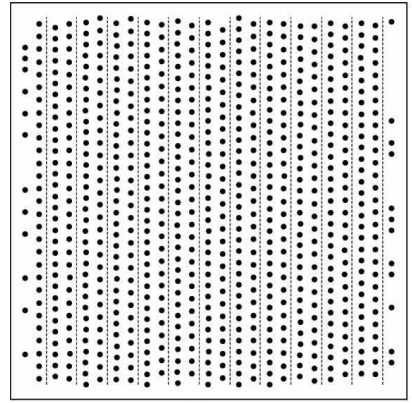

(a)

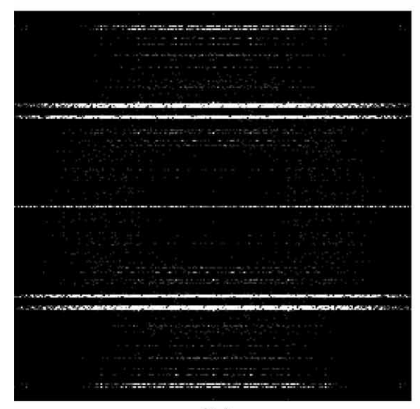

(b)

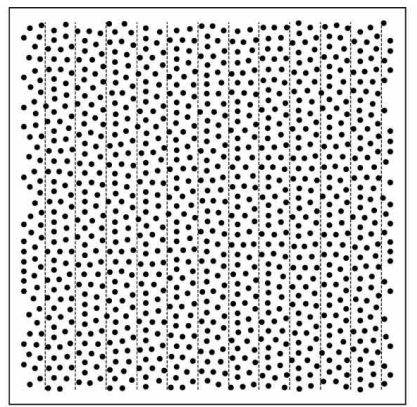

(c)

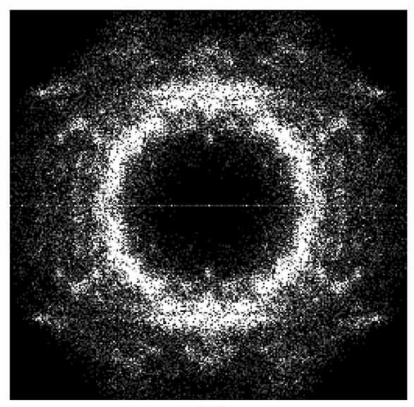

(d)

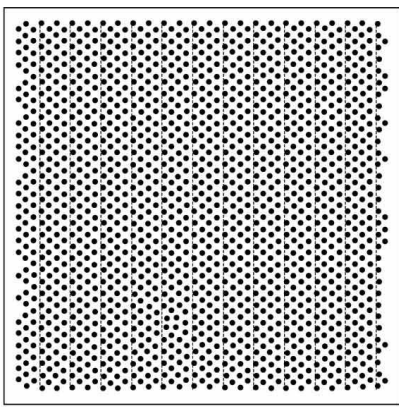

(e)

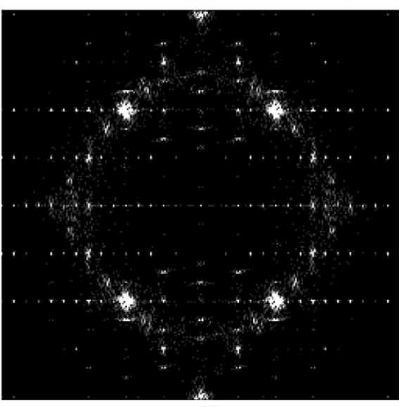

(f)

FIG. 6: (a) Vortex locations (black dots) and edges of the pinning troughs (black lines) in a $12 \lambda \times 12 \lambda$ subsection of a system with $n_{v}=5.21 / \lambda^{2}$ and no applied drive. Each trough captures two columns of vortices. (b) Corresponding $S$ (k) for $n_{v}=5.21 / \lambda^{2}$ showing evidence of smectic ordering. (c) Vortex and pinning trough locations for $n_{v}=6.94 / \lambda^{2}$. The vortex configurations are disordered. (d) $S(\mathbf{k})$ for $n_{v}=6.94 / \lambda^{2}$ has a clear ring structure. (e) Vortex and pinning trough locations for $n_{v}=10.42 / \lambda^{2}$. The vortices form a tilted square lattice. (f) $S(\mathbf{k})$ for $n_{v}=10.42 / \lambda^{2}$ shows four-fold peaks.

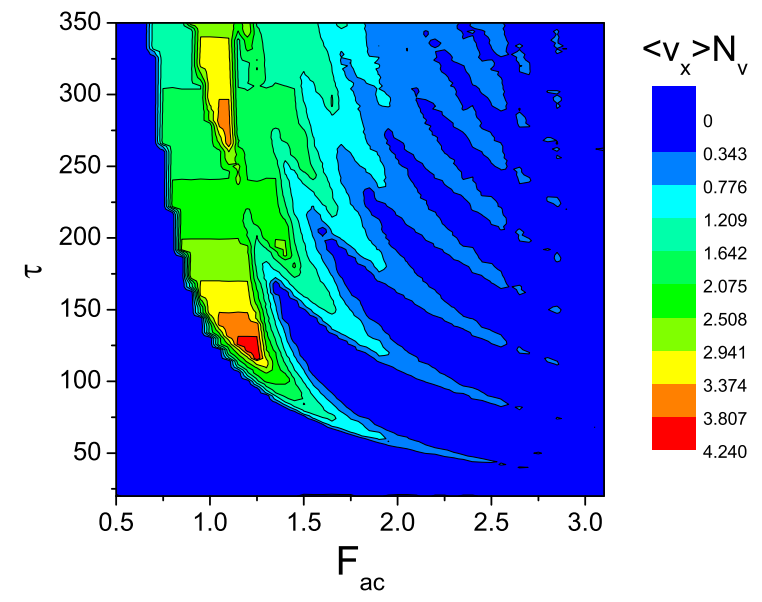

FIG. 7: (Color online) Contour plot of $\left\langle V_{x}\right\rangle$ as a function of ac drive period $\tau$ (in units of simulation time steps) and ac drive amplitude $F_{a c}$ (in units of $f_{0}$ ) for a system with $n_{v}=0.087 / \lambda^{2}$.

substrates, where crystalline, smectic, and partially disordered structures were observed as a function of particle density [19]. The double smectic phases and the square lattice that we find did not appear in the magnetic particle experiment since the relative particle densities considered in Ref. [19] were much lower than the relative vortex densities we use here. Additionally, the asymmetry in our potential substrate may help to stabilize certain phases that are not seen with simple symmetric potentials.

\section{RATCHET AND REVERSE RATCHET EFFECT}

We next examine the response when an external ac drive is applied. When the vortex density is low, the vortex-vortex interactions are negligible and the system responds in the single particle limit. In Fig. 7 we show a contour plot of the average velocity $\left\langle V_{x}\right\rangle$ obtained from simulations with $n_{v}=0.087 / \lambda^{2}$ for varied ac period $\tau$ and ac amplitude $F_{a c}$. A series of tongues where a positive ratchet effect occurs are clearly visible. At small $\tau$, there is not enough time for the vortices to respond to the ac drive so there is no ratchet effect. Similarly, at low $F_{a c}$, the vortices are unable to cross the potential barrier of the trough, and no rectification occurs. At intermediate values of $\tau$ and $F_{a c}$, a ratchet effect can occur. In the first tongue, which falls at the lower left edge of Fig. 7 , the vortices move by $+a$ in the $x$ direction during the positive half of the driving period, but do not move back during the negative half of the period, resulting in a net 


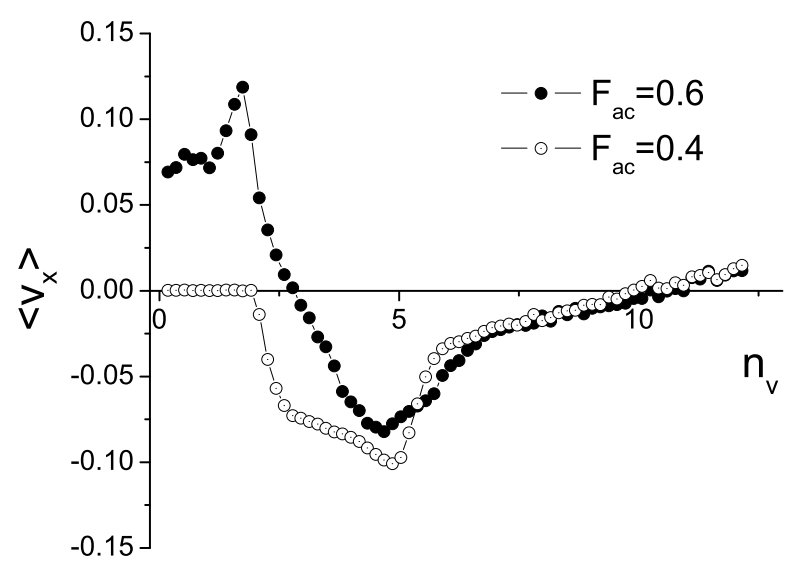

FIG. 8: $\left\langle V_{x}\right\rangle$ vs $n_{v}$ for the system in Fig. [7 at fixed $\tau=10^{4}$ simulation steps for (black circles) $F_{a c}=0.6 f_{0}$ and (open circles) $F_{a c}=0.4 f_{0}$. Here both positive and negative ratchet effects occur as a function of vortex density.

rectification. On the second tongue, vortices translate by $+2 a$ during the first half of the period and by $-a$ during the second half of the period, again giving a net rectification. As $\tau$ and $F_{a c}$ increase, the ratchet effect on the $n$th tongue is characterized by motion of $+n a$ during the positive half period of the drive and motion of $-(n-1) a$ during the negative half period of the drive. The tongue structure emerges since for some values of $\tau$ and $F_{a c}$, vortices undergo equal translations during both halves of the driving period, leading to no net motion. The boundary of the $n$th tongue where ratcheting can occur is given by

$$
\tau_{n}=\eta \frac{n l^{+}}{F_{a c}-F^{+}}+\eta \frac{(n-1) l^{-}}{F_{a c}+F^{-}}
$$

The weak pinning force $F^{+}$associated with the trough side of length $l^{+}$is $F^{+}=-A_{p} / 2$ in our case, while the strong pinning force $F^{-}$associated with the trough side of length $l^{-}$is $F^{-}=A_{p}$. For the first tongue in Fig. 7 . Eq. 4 predicts $\tau_{1} \propto\left(F_{a c}+A_{p} / 2\right)^{-1}$, which matches the simulation results. The results in Fig. 7 and Eq. 4 agree well with the tonguelike features found in Ref. [5] as a function of $\tau$ and $F_{a c}$. This confirms that at low vortex densities, an ordinary ratchet effect occurs where the vortices move in the easy direction.

We next consider the effect of increasing $n_{v}$. Since the ratchet effect in Fig. 7 is enhanced for larger periods $\tau$, we fix the period at $\tau=10^{4}$ simulation steps. In Fig. 8 we plot $\left\langle V_{x}\right\rangle$ versus $n_{v}$ for $F_{a c}=0.6 f_{0}$ and $0.4 f_{0}$. For $n_{v}<2.60 / \lambda^{2}$, there is a positive ratchet effect for $F_{a c}=0.6 f_{0}$ and no ratchet effect for $F_{a c}=$ $0.4 f_{0}$. For $n_{v}>2.60 / \lambda^{2}$ a negative ratchet effect occurs for $F_{a c}=0.4 f_{0}$, while the positive ratchet effect for $F_{a c}=0.6 f_{0}$ decreases in size and reverses to a negative ratchet effect near $n_{v}=3.65 / \lambda^{2}$. For both values of $F_{a c}$,

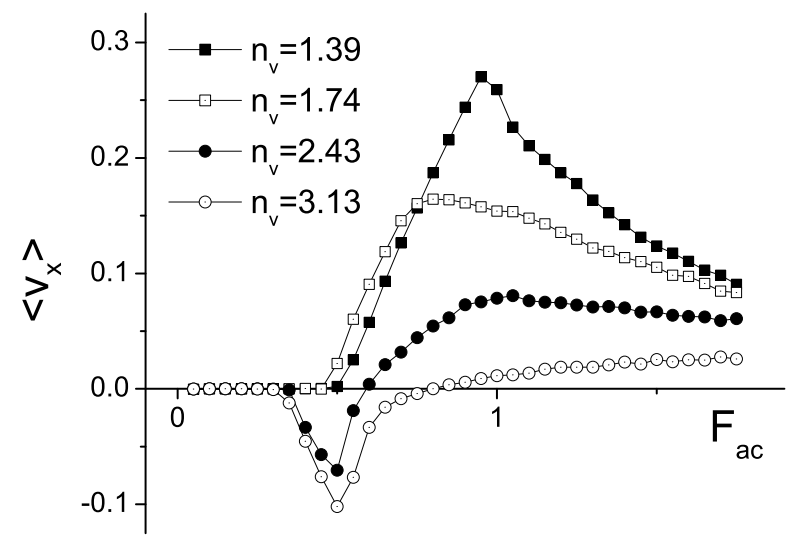

FIG. 9: $\left\langle V_{x}\right\rangle$ vs $F_{a c}$ for the same system as in Fig. 8 for varied $n_{v}$. Filled squares: $n_{v}=1.39 / \lambda^{2}$; open squares: $n_{v}=1.74 / \lambda^{2} ;$ filled circles: $n_{v}=2.43 / \lambda^{2}$; open circles: $n_{v}=3.13 / \lambda^{2}$. The positive ratchet effect is preceded by a negative ratchet effect for $n_{v}>1.74 / \lambda^{2}$.

the system passes through a maximum negative value of $\left\langle V_{x}\right\rangle$ just below $n_{v}=5.21 / \lambda^{2}$. Above $n_{v}=5.21 / \lambda^{2}$, $\left\langle V_{x}\right\rangle$ gradually increases back toward zero before crossing zero and reversing to a small positive ratchet effect near $n_{v}=10.42 / \lambda^{2}$. The features in these curves appear to be correlated with the vortex lattice structures presented in Figs [5] and [6. At low $n_{v}$, the vortices form a triangular or smectic structure with only a single column of vortices in each pinning trough, as in Fig. $5(a, b)$. In this case, since the symmetry of the vortex configuration cancels or strongly reduces the vortex-vortex interaction force, a positive ratchet effect occurs that is determined only by the pinning forces of the substrate. The onset of the negative ratchet effect corresponds to the appearance of buckling of the columns of vortices in the pinning troughs, as seen in Fig. [5(c,d). In the double smectic phase illustrated in Fig. $5(e, f)$ and Fig. $6(a, b)$, the negative ratchet effect persists. The gradual disappearance of the negative ratchet effect for $n_{v}>5.21 / \lambda^{2}$ in Fig. 8 is associated with the crossover from the double smectic state to the disordered state shown in Fig. 6(c,d). The small positive ratchet effect near $n_{v}=10.42 / \lambda^{2}$ is correlated with the formation of the ordered square lattice as seen in Fig. [6 $(e, f)$.

In Fig. 9 we plot $\left\langle V_{x}\right\rangle$ versus $F_{a c}$ for a series of simulations performed at varied $n_{v}$. For low $n_{v} \leq 1.74 / \lambda^{2}$, there is no ratchet effect until a threshold value of $F_{a c}$ is reached. $\left\langle V_{x}\right\rangle$ then increases to a peak value and gradually decreases with increasing $F_{a c}$. For $n_{v}>1.74 / \lambda^{2}$, the threshold value of $F_{a c}$ above which a ratchet effect occurs is lower than for $n_{v} \leq 1.74 / \lambda^{2}$, and the initial ratchet effect is in the negative direction rather than the positive direction. As $F_{a c}$ is increased, the negative ratchet effect reverses to a positive ratchet effect.

Figure 10 illustrates $\left\langle V_{x}\right\rangle$ in a contour plot as a func- 


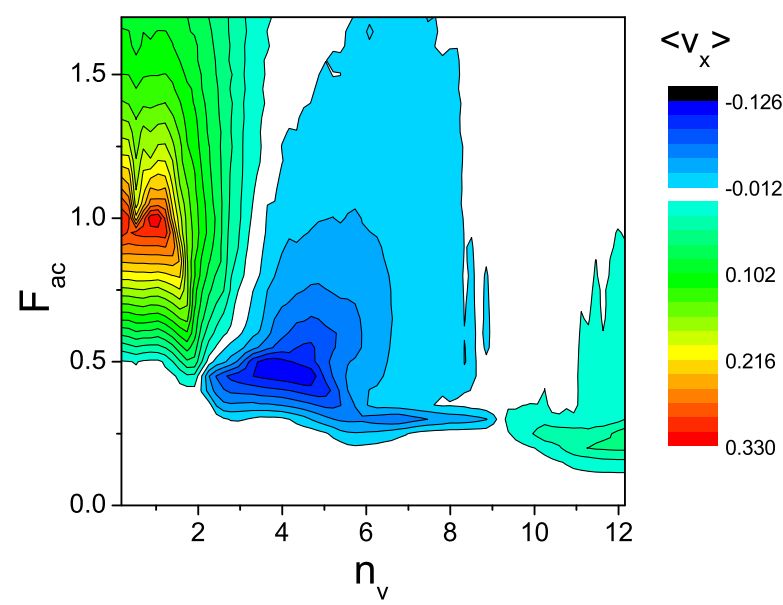

FIG. 10: (Color online) A colorscale map of $\left\langle V_{x}\right\rangle$ as a function of $F_{a c}$ and $n_{v}$. For low $F_{a c}$ there is no ratchet effect. At higher $F_{a c}$ there is a positive ratchet effect for low $n_{v}$, a reversal to a negative ratchet effect for intermediate $n_{v}$, and another reversal at high $n_{v}$ to a positive ratchet effect.

tion of $F_{a c}$ and $n_{v}$. The positive and negative ratchet effect regimes are highlighted. For low $F_{a c}$ there is no ratchet effect, while for high $F_{a c} / f_{0}>1.0$ all ratchet effects are gradually reduced. The maximum positive ratchet effect occurs for $F_{a c} / f_{0}$ close to 1.0 , the value of the pinning force in the strong direction. The positive ratchet effect for $n_{v}<2.60 / \lambda^{2}$ does not occur unless $F_{a c} / f_{0}>0.5$, which is the maximum pinning force in the weak direction. The negative ratchet effect appears for $n_{v}>2.08 / \lambda^{2}$ and extends up to $n_{v}=8.68 / \lambda^{2}$, with the maximum negative rectification occurring for $3.47 / \lambda^{2}<n_{v}<5.21 / \lambda^{2}$ at $0.45<F_{a c} / f_{0}<0.5$. For $n_{v}>9.72 / \lambda^{2}$, a reentrant positive ratchet effect appears that is much weaker in amplitude than the positive ratchet effect region found for low $n_{v}$. In general, the ratchet effect in either direction is reduced for increasing $n_{v}$ since the effects of the substrate are gradually washed out by the increasing vortex-vortex interaction forces. This result shows that vortices interacting with simple asymmetric pinning potentials can exhibit multiple ratchet reversals similar to those seen for the more complex 2D pinning array geometries [17]. It is likely that for higher $n_{v}$, higher order reversals will occur when the vortices again form a smectic phase with five or more vortex chains per pinning trough. We are not able to access the high vortex densities where these reversals would be expected to appear. The higher order ratchet effects would likely continue to decrease in amplitude with increasing field.

The positive or regular ratchet effect that occurs when the vortices move in the easy direction is straightforward to understand at low fields based on a single particle pic-

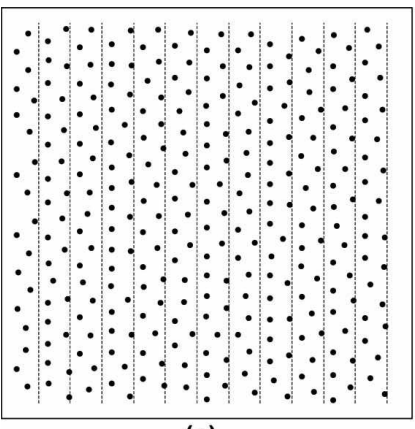

(a)

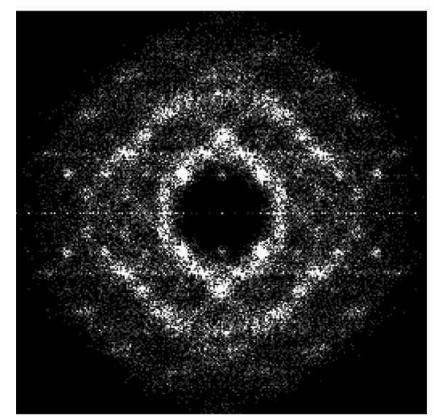

(b)

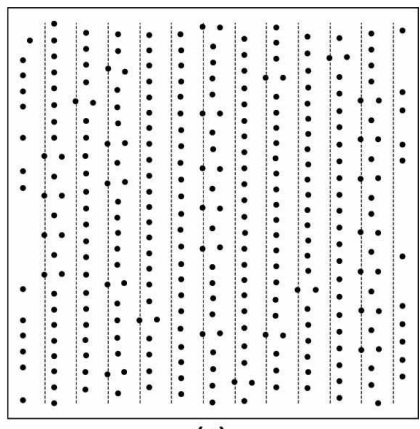

(c)

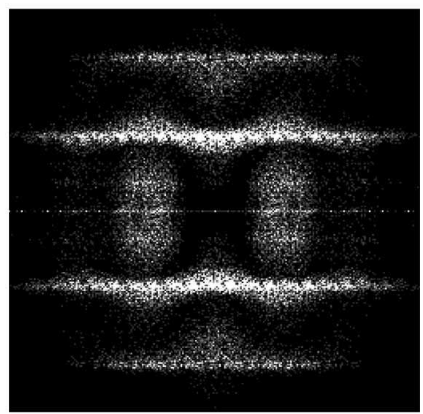

(d)
FIG. 11: (a) Vortex locations (black dots) and edges of the pinning troughs (black lines) in a $12 \lambda \times 12 \lambda$ subsection of a system with $n_{v}=2.08 / \lambda^{2}$ at $F_{a c}=0.5 f_{0}$ when the negative ratchet effect occurs. The positive portion of the driving period, with $\mathbf{F}_{i}^{e x t}=\hat{\mathbf{x}} F_{a c}$, is shown. The vortices form two columns in every trough. (b) The corresponding structure factor $S(\mathbf{k})$ for positive drive. The overall disorder in the vortex configuration is indicated by the ring structure. (c) Vortex and pinning trough locations for the same system during the negative portion of the driving period, with $\mathbf{F}_{i}^{e x t}=-\hat{\mathbf{x}} F_{a c}$. The vortices are arranged in buckled columns. (d) The corresponding $S(\mathbf{k})$ for negative drive shows a smectic type of ordering.

ture. The first reversal of the ratchet effect for increasing $N_{v}$ is related to the vortex-vortex interactions. This is more clearly seen from Fig. 11, where we plot the vortex positions and corresponding structure factor during both halves of the driving cycle for $n_{v}=2.08 / \lambda^{2}$ and $F_{a c}=0.5 f_{0}$ where a negative ratchet effect occurs. During the positive drive portion of the ac cycle, the vortices are roughly evenly spaced in a disordered arrangement, as shown in Fig. 11( $a, b)$. When the negative drive is applied, the vortices form a clear smectic structure with roughly one vortex column per pinning trough, as illustrated in Fig. 11 (c,d). In this case, the vortex configuration is asymmetric due to the appearance of buckling in the vortex columns. At a buckled location, an extra vortex appears on the positive $x$ side of the vortex column (to the right of the column) in the same trough. These extra vortices effectively push the adjacent vortices in the column over the potential barrier in the hard or negative $x$ direction.

If the extra vortex is located a distance $a$ from the 
column, then a portion of the vortices in the column can escape over the potential barrier in the hard direction when the following condition is met:

$$
F_{a c}+K_{1}(a) f_{0}>A_{p}
$$

When $A_{p} / f_{0}=1.0$ and $K_{1}(a)=0.6$, a negative ratchet effect is predicted to occur when $F_{a c} / f_{0}>0.4$, which is what is observed in Fig. 9. It could also be argued that when the drive is in the positive direction, the extra vortices should be pushed by the column of vortices and should more easily move in the positive direction, allowing the conditions of Eq. 5 to be met in the positive direction as well. In Fig. 11(a) we show that this does not occur because the vortex arrangement is quite different during the positive drive portion of the cycle. The vortex-vortex interaction term is not present since the vortices do not form the smectic structure that is seen when the driving is in the negative direction. Instead, the vortices form two columns within each pinning trough, allowing the individual vortices to remain evenly separated in both the $x$ and $y$ directions, and preventing an individual vortex from pushing another vortex out of the trough. The vortex structure for positive drive is more symmetrical, as seen in $S(\mathbf{k})$ in Fig. 11(b) which has a smeared crystalline or disordered structure. This symmetry reduces the vortex-vortex interaction forces.

A ratchet effect in the positive direction occurs when the following equation is satisfied:

$$
F_{a c}>A_{p} / 2 .
$$

If $F_{a c} / f_{0}=0.45$ and $A_{p} / f_{0}=1.0$, then the conditions of Eq. 5 are met but the conditions of Eq. 6 are not, so the negative ratchet effect can occur but not the positive ratchet effect. Once $F_{a c} / f_{0}>0.5$, it is also possible for the positive ratchet effect to occur and the two ratchet mechanisms will compete, decreasing the magnitude of the negative ratchet effect as seen in the phase diagram of Fig. 10 for higher $F_{a c}$. As $n_{v}$ is increased, the smectic structure is gradually lost as shown in Fig. 6(c,d), so the vortex-vortex interactions required to produce the negative ratchet effect are reduced and the negative ratchet effect is diminished. Once the vortex lattice is again symmetrical, as in Fig. 6(e,f), the positive ratchet effect appears. This result suggests that in regions where a reversed ratchet effect appears, the vortex lattice structure must exhibit some asymmetry when driven in the hard direction for the reverse ratchet effect to occur.

We have also investigated the reversal of the ratchet effect as a function of the pinning strength $A_{p}$ for fixed $n_{v}=3.13 / \lambda^{2}$ and $\tau=10^{4}$. In Fig. 12 we plot $\left\langle V_{x}\right\rangle N_{v}$ versus $A_{p}$ for $F_{a c}$ ranging from $0.4 f_{0}$ to $0.8 f_{0}$. For all values of $F_{a c}$, as $A_{p}$ increases a positive ratchet effect appears. This is because the stronger $A_{p}$ forces the vortices to form completely 1D unbuckled columns inside the pinning troughs. For lower $A_{p}$, the vortex columns can buckle, providing the unbalanced vortex-vortex interactions required to produce the negative ratchet effect.

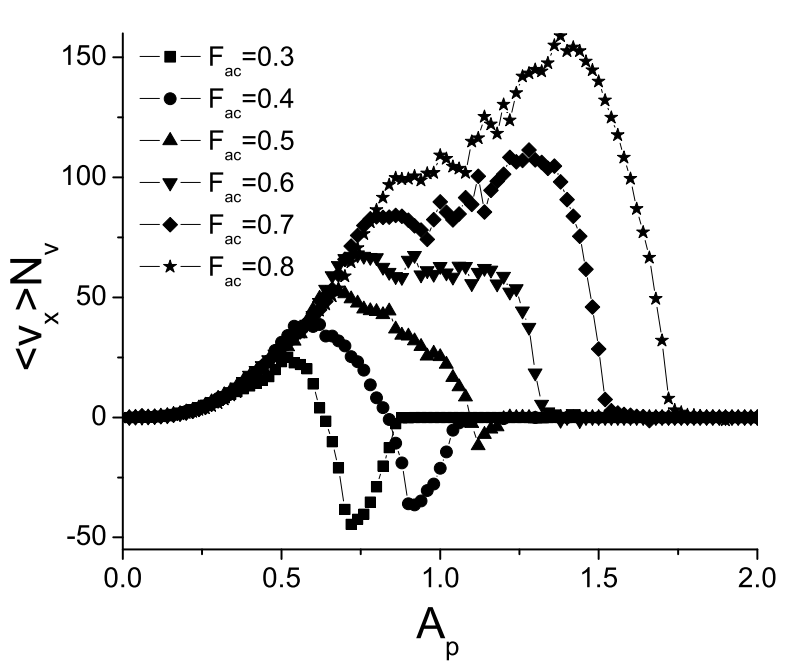

FIG. 12: $\left\langle V_{x}\right\rangle N_{v}$ vs $A_{p}$ for the same system as in Fig. 10 with $n_{v}=3.13 / \lambda^{2}$. Squares: $F_{a c}=0.3 f_{0}$; circles: $F_{a c}=$ $0.4 f_{0}$; up triangles: $F_{a c}=0.5 f_{0}$; down triangles: $F_{a c}=0.6 f_{0}$; diamonds: $F_{a c}=0.7 f_{0}$; stars: $F_{a c}=0.8 f_{0}$. For large $A_{p}$ only a positive vortex ratchet effect occurs as the vortices form a single column in each pinning trough. As the pinning strength is reduced, buckling of the columns begins to occur and a negative ratchet effect appears.

The negative rectification is enhanced for both low $A_{p}$ and low $F_{a c}$. Changing the strength of $A_{p}$ in a single superconducting sample is difficult; however, a geometry similar to that shown in Fig. 1 could be created using tunable optical traps for colloidal particles driven with an ac electric field.

\section{SUMMARY}

In conclusion, we have shown that the vortex ratchet system proposed by Lee et al. in Ref. [5] for vortices interacting with simple periodic asymmetric pinning substrates can exhibit a series of ratchet reversals similar to those observed for 2D asymmetric periodic pinning arrays. Unlike most vortex ratchet geometries considered to date, the ratchet effects presented here can only be explained by a $2 \mathrm{D}$ description of the vortex configuration, and not by a simple 1D model. As a function of vortex density, a rich variety of vortex phases can be realized in this system including triangular, smectic, disordered, and square lattices. The dc critical depinning forces exhibit a reversible diode effect as a function of vortex density. At low magnetic fields, the depinning force is higher in the hard direction, corresponding to the regular diode effect, while at higher magnetic fields, the depinning force is lower in the hard direction. Another reversal of the diode effect occurs at even higher fields. The reversed diode effect appears when the symmetry of the vortex configuration changes for different directions of dc drive, as in 
the case of the smectic and disordered phases. Here, the importance of vortex-vortex interactions differs for the two drive directions. At densities where the vortex lattice is highly ordered, a regular diode effect occurs where the vortex depinning force is lower in the easy direction. The vortex lattice structures are also directly correlated with the sign of the ratchet effect that occurs under an ac drive. The highly symmetric structures ratchet in the easy direction since the vortex-vortex interactions effectively cancel, causing the sign of the ratchet effect to be determined only by the asymmetry of the pinning substrate. In the smectic states, the vortex-vortex interactions become relevant and can produce a negative ratchet effect, which can be predicted with force balance arguments. We have shown the dependence of the reversible ratchet effect on ac amplitude, vortex density, and pinning strength. This type of ratchet could be also realized for other kinds of systems composed of collections of repulsively interacting particles and simple asymmetric substrates, such as colloid systems.

\section{ACKNOWLEDGMENTS}

This work was carried out under the auspices of the National Nuclear Security Administration of the U.S. Department of Energy at Los Alamos National Laboratory under Contract No. DE-AC52-06NA25396. Q.L. was also supported in part by NSF Grant No. DMR-0426488.
[1] For a review, see: P. Reimann, Phys. Rep. 361, 57 (2002).

[2] S.H. Lee, K. Ladavac, M. Polin, and D.G. Grier, Phys. Rev. Lett. 94, 110601 (2005); A. Libál, C. Reichhardt, B. Jankó, and C.J. Olson Reichhardt, Phys. Rev. Lett 96, 188301 (2006).

[3] Z. Farkas, P. Tegzes, A. Vukics, and T. Vicsek, Phys. Rev. E 60, 7022 (1999); J.F. Wambaugh, C. Reichhardt, and C.J. Olson, ibid. 65, 031308 (2002).

[4] T.S. Monteiro, P.A. Dando, N.A.C. Hutchings, and M.R. Isherwood, Phys. Rev. Lett. 89, 194102 (2002); E. Lundh and M. Wallin, ibid. 94, 110603 (2005).

[5] C.S. Lee, B. Jankó, I. Derényi, and A.L. Barabási, Nature (London) 400, 337 (1999).

[6] J.F. Wambaugh, C. Reichhardt, C.J. Olson, F. Marchesoni, and F. Nori, Phys. Rev. Lett. 83, 5106 (1999).

[7] C.J. Olson, C. Reichhardt, B. Jankó, and F. Nori, Phys. Rev. Lett. 87, 177002 (2001).

[8] J. Van de Vondel, C.C. de Souza Silva, B.Y. Zhu, M. Morelle, and V.V. Moshchalkov, Phys. Rev. Lett. 94, 057003 (2005); C.C. de Souza Silva, J. Van de Vondel, B.Y. Zhu, M. Morelle, and V.V. Moshchalkov, Phys. Rev. B 73, 014507 (2006).

[9] Y. Togawa, K. Harada, T. Akashi, H. Kasai, T. Matsuda, F. Nori, A. Maeda, and A. Tonomura, Phys. Rev. Lett. 95, 087002 (2005).

[10] T.C. Wu, L. Horng, J.C. Wu, C.W. Hsiao, J. Kolacek, and T.J. Yang, J. Appl. Phys. 99, 08M515 (2006).

[11] D.Y. Vodolazov, F.M. Peeters, I.V. Grigorieva, and A.K. Geim, Phys. Rev. B 72, 024537 (2005);
D.Y. Vodolazov and F.M. Peeters, ibid. 72, 172508 (2005).

[12] C. Reichhardt, C.J. Olson, and M.B. Hastings, Phys. Rev. Lett. 89, 024101 (2002); C. Reichhardt and C.J. Olson Reichhardt, Physica C 404, 302 (2004).

[13] M.B. Hastings, C.J. Olson Reichhardt, and C. Reichhardt, Phys. Rev. Lett. 90, 247004 (2003).

[14] B.Y. Zhu, F. Marchesoni, V.V. Moshchalkov, and F. Nori, Phys. Rev. B 68, 014514 (2003); B.Y. Zhu, F. Marchesoni, and F. Nori, Phys. Rev. Lett. 92, 180602 (2004).

[15] J.E. Villegas, S. Savel'ev, F. Nori, E.M. Gonzalez, J.V. Anguita, R. Garcia, and J.L. Vicent, Science 302, 1188 (2003).

[16] C.J. Olson Reichhardt and C. Reichhardt, Physica C 432, 125 (2005).

[17] C.C. de Souza Silva, J. Van de Vondel, M. Morelle, and V.V. Moshchalkov, Nature (London) 440, 651 (2006).

[18] D.E. Shalom and H. Pastoriza, Phys. Rev. Lett. 94, 177001 (2005); V.I. Marconi, Physica C 437, 195 (2006); V.I. Marconi, to be published.

[19] J. Hu and R.M. Westervelt, Phys. Rev. B 55, 771 (1997).

[20] C. Reichhardt, C.J. Olson, J. Groth, S. Field, and F. Nori, Phys. Rev. B 52, 10441 (1995).

[21] S.H. Brongersma, E. Verweij, N.J. Koeman, D.G. de Groot, R. Griessen, and B.I. Ivlev, Phys. Rev. Lett 71, 2319 (1993). 